\title{
REVERSE ENGINEERING APPLICATIONS IN MANUFACTURING INDUSTRIES: AN OVERVIEW
}

\author{
RATHORE, N. \& JAIN, P. K.
}

Abstract: With the evolving needs of today's manufacturing industries in the area of product development, 3D CAD model has become very important. Often in many situations there is a need to remanufacture a physical part where the CAD data is not available with the manufacturer or is lost because of several reasons. In such cases reverse engineering is found to be an effective and efficient tool. Reverse engineering is an approach where the physical parts are digitized in order to obtain a virtual model. Digitization can be done either manually or it can be fully automatized for better and fast scans. The virtual models so obtained are helpful in many applications but often used for redesign operations. This paper reviews the reverse engineering process and its application in various manufacturing industries. After a brief introduction, methodology used in reverse engineering and its applications in different fields have been discussed. Future scope of RE is also discussed in this paper.

Key words: reverse engineering, optical $3 D$ digitization, scanned data, recreating, manufacturing industries
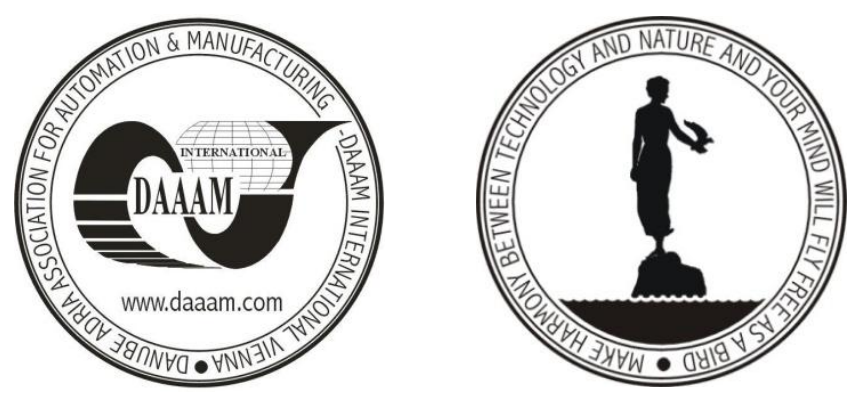

Authors' data: Rathore, N[ikki]; Jain, P[ramod] K[umar], Mechanical \& Industrial Engineering Department, Indian Institute of Technology, Roorkee, 247667, Uttarakhand India, nikkirathore2012@gmail.com, pjainfme@iitr.ernet.in

This Publication has to be referred as: Rathore, N[ikki] \& Jain, P[ramod] K[umar] (2014). Reverse Engineering Applications in Manufacturing Industries: an Overview, Chapter 45 in DAAAM International Scientific Book 2014, pp.567-576, B. Katalinic (Ed.), Published by DAAAM International, ISBN 978-3-901509-98-8, ISSN 17269687, Vienna, Austria

DOI: 10.2507/daaam.scibook.2014.45 
Rathore, N. \& Jain, P. K.: Reverse Engineering Applications in Manufacturing Indu...

\section{Introduction}

Due to the competitive nature of today's market, manufacturers face a tough race. In order to win the race they should remain a step ahead of others for which the product demand's a short lead time and better features. In such an inconsistent domain one needs to minimize the loss by reducing the waste. To do so lean manufacturing should be adapted by the manufactures. For this conventional methods of manufacturing are not efficient because these results in work in process inventory and are not suitable for producing small orders. Process like reverse engineering and rapid prototyping help us to solve some of such problems.

Reverse engineering is defined as a process in which a CAD model of a physical part is obtained in order to remanufacture it or to reveal its design secrets. RE may also be defined as the "process of retrieving new geometry from a manufactured part by digitizing and modifying an existing CAD model" (Yau, 1997). Reverse Engineering (RE) may also be referred as "the basic concept of producing a part based on an original or physical model without the use of an engineering drawing" (Abella, 1994).

Now a day's reverse engineering has various fields of application but to create an old artifact, it might be the only technique available. In research work one can use it to create a 3- dimensional CAD model from a physical part which is made up of clay and then the model can be easily modified according to the needs of research work in a much faster manner. Sometimes at shop floor changes have been incorporated in the product/part or tool to meet the quality requirements and also to increase manufacturability, these changes are not included in the actual CAD model of the part. By using reverse engineering tool, production drawings can be updated and same can used for next production run. Hence RE is applicable to a variety of fields in manufacturing industries.

\section{Reverse engineering process}

Reverse engineering process contains three major steps which are used to extract knowledge about the design, material, structure, surface qualities, and working conditions of the product etc. The procedural steps are defined as scanning phase, point processing phase and solid modeling. M. Chang (2008) further categorized these steps as data acquisition, preprocessing (noise filtering and merging), triangulation, feature extraction, and segmentation and surface fitting. Fig.1 shows the working process of RE. The whole process of reverse engineering should be computer aided.

Before reverse engineering a part few points must be considered in planning strategy such as reasons for which a part is to be reverse engineered (documentation is lost or to duplicate competitors product etc.), part size (small or large), part complexity (simple or complex), part material (hard or soft), part geometry (organic or prismatic, internal or external), part finish (shiny or dull), number of parts to be scanned (single or multiple), accuracy required (volumetric or linear) etc. 


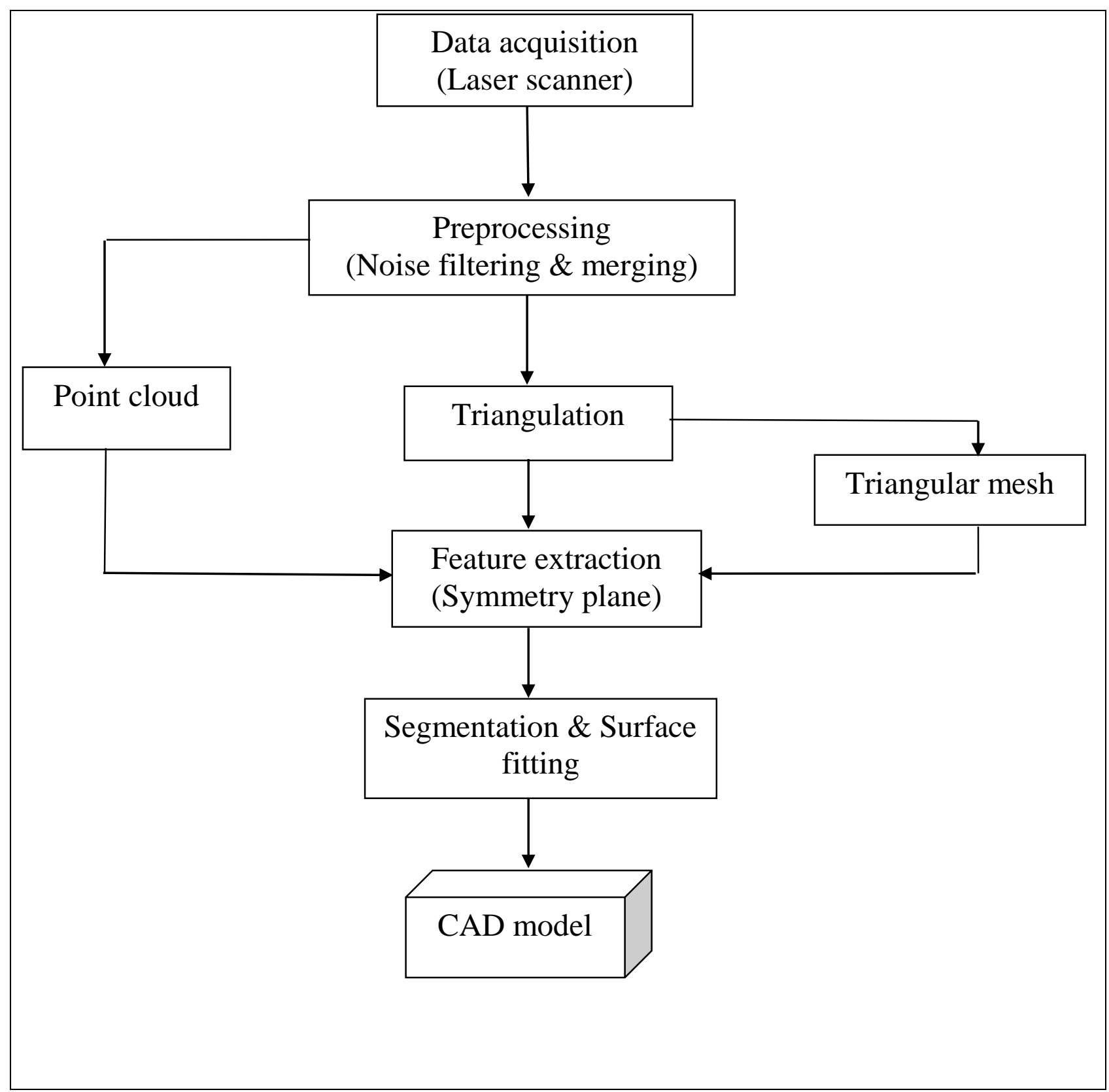

Fig. 1. Flow of reverse engineering process (Chang, 2008)

The whole process of RE is discussed in detail as follows:

1. Scanning/ Data Acquisition: This phase is intended to gather the data about the geometric aspect of the physical part through scanning. In this stage physical part which is to be recreated is prepared for scanning and then actual scanning is performed using a suitable scanner(laser or CMM) in order to seize all the information regarding the physical features of the part e.g. holes, slots, curves, steps etc. In general there are two types of scanners contact type and noncontact type. Contact scanners are those which contain a contact probe which moves along the silhouette of physical part. Common example of such a scanner is co-ordinate measuring machine (CMM) while the non-contact types are the one which do not have a contact probe instead they uses lasers, optics, and charge-coupled device (CCD) sensors to capture the data. 
Rathore, N. \& Jain, P. K.: Reverse Engineering Applications in Manufacturing Indu...

2. Preprocessing/Point Processing Phase: In this phase obtained data as a result of scanning is processed in order to remove the noise and to reduce the number of data points. Different types of filters are available to reduce the cloud points. This phase also gives us the advantage to merge multiple scan data sets. Sometimes multiple scans are performed on the part to make certain that all features are scanned. For this a part is rotated, hence it becomes necessary to plan a good datum so that the merging of data sets can be done successfully which will eventually reduce the occurrence of error in point data. A number of commercial software's are available for point processing.

3. Triangulation and Feature extraction: Triangulation is a process of formation or division of point data into triangles. In this step triangulation of point data is carried out by creation of triangular mesh using a suitable algorithm. Feature extraction is the next step in this phase which is defined as the process of defining a set of features, or image characteristics, which will most efficiently represent the information that is important for analysis and classification.

4. Segmentation and Surface fitting, and Solid Modeling: This is known as the most complex stage of reverse engineering as dynamic fitting algorithms are used for surface generation that flawlessly represent the 3D information depicted within the point cloud data sets. Segmentation is the most critical step in reverse engineering. Segmentation is defined as the process of splitting a triangular mesh into sub-meshes to which an appropriate single surface can be fitted which seriously affects the quality of the resulting CAD model and then the 3 dimensional CAD model of the physical part is obtained. Most CAD systems are not designed to display and process large amounts of point data, as a result new RE modules or discrete software packages are generally needed for point processing.

\section{Historical background}

A huge amount of literature is available in journals and books which explains the process of reverse engineering and its applications in the various fields. Here, a review of significant literature has been made. The overview of literature was based on the applications of Reverse Engineering in automobile industry, automotive industry, medical life sciences and software industries. Many researchers have experimented in this regard.

E. Bagci (2009) defined the reverse engineering process as the five stage process which includes digitization, data capturing, processing phase, surface approximation (for solid modeling), technical documentation and, NC part programming and $\mathrm{CNC}$ milling machine ( for the part manufacturing) using three samples which were broken and worn in some regions. Components used for study are damaged cam-worn due to friction, turbine blade and a defected Ataturk's bust. These components were recreated using RE and modifications have been made to them in order to recover their final geometry. After recovery CAD models were used for part manufacturing using a CNC milling machine. Fig. 2 describes the process and end application of reverse engineering.

$\mathrm{RE}$ is applied to manufacturing industries in regards to following reasons: 
1. Original design data of the part is not available e.g. in case of military applications.

2. The original producer no longer produces the product. Such situations can be seen in electronics, automotive, aerospace industries.

3. Also helpful in repairing a damaged part by comparing the CAD data of worn out part to its actual CAD design data e.g. damaged turbine blades now-a-days are generally repaired using reverse engineering approaches.

4. It also reduces the research and development cycle time for a new artifact based on an existing product by significantly reducing the model building cycle, and prototyping costs.

5. It can be used to gain competitive benchmarking by analyzing competitors part to reveal its secret.

6. To speed up innovation in areas such as ergonomic design, retro-inspired design, combustion design, and aerodynamics, where CAD modeling often adds an unnecessary step.

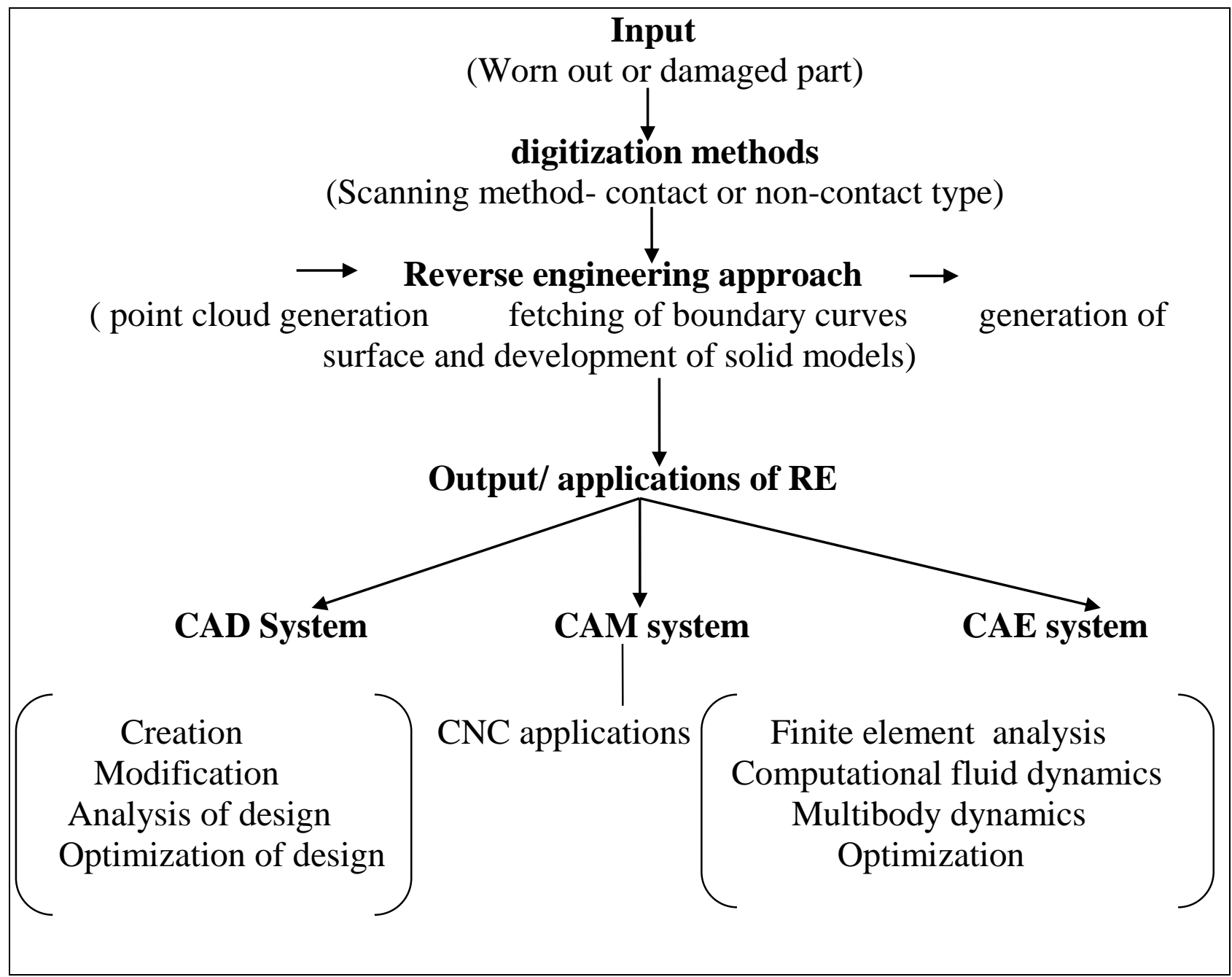

Fig. 2. Applications of reverse engineering

\subsection{Applications in mechanical industries}

In mechanical industry one can use reverse engineering in order to fulfill following requirements: 
Rathore, N. \& Jain, P. K.: Reverse Engineering Applications in Manufacturing Indu...

1. For creating 3D model of a physical part with lost documentation.

2. For keeping a digital 3D record of own products.

3 . For assessing competitor's products.

4. To analyze the working of a product.

5. To inspect and compare actual geometry with CAD model.

6. To measure wear of tools.

Y. Zang (2003) had described the RE process from digitization, CAD model recreation to $\mathrm{NC}$ machining by successfully creating a 3D CAD model of a core die of the inlet of a diesel engine. The model was then used to generate machining codes through $\mathrm{NC}$ post-processing for the manufacturing of the die using a $\mathrm{CNC}$ milling machine.

G. Sansoni and F. Docchio (2004) have demonstrated the use of reverse engineering technique for large surfaces with high accuracy and reduced processing time, for design and restyling applications. In their research a former automobile, a Ferrari 250 Mille Miglia using a 3D digitizer which was based on the projection of incoherent light (OPL- 3D, developed by them) was reverse engineered and then remanufactured by RP. Process includes the alignment of point cloud data, formation of triangulated mesh, the NURBES creation, formation of STL file and at last scaled replica of the car was generated.

J. Gao et.al. (2006) said that no two mechanical parts are identical after definite interval of working due to wear/ damages. So the CAD data of worn out part is different from designed CAD data as a result designed data cannot be used for tool path generation for repair processes. Hence they have proposed a repair strategy using a worn out blade of turbine generator to generate correct tool paths suitable to each worn out part through reverse engineering. They have also defined a polygon modeling technique based on 3D scanning to repair parts for direct use of manufacturing process like machining, welding and inspection.

H. Wu et.al. (2013) have reviewed several algorithms based on geometric reconstruction and repairing methodologies for gas turbine component which is a critical component because of its complexity in shape and high cost of repair. The paper includes different types of algorithms for the repairing solutions of damaged blades. The process of repair and overhaul is a five stage process: pre repair inspection, identification, surface reconstruction of defected surface, welding, milling and grinding. They also concluded that available reconstructing approaches are not efficient so future work is required in this field.

S. G. Lee et.al. (2008) have worked on the working processes of maintenance, repair and overhaul in aviation industry by reviewing the characteristics and benefits of product lifecycle and management (PLM) using two case studies. They have also discussed the evolution of CAD, CAM and CAE tools through product data management systems. PLM evolved from two streams one is enterprise management and the other is supply chain management. In first case study they concluded that because of less share of information between OEM and downstream repair 
companies, repair processes cannot be done accurately because jigs and fixtures designed for repair purposes were based on the virtual model created using a damaged part and not on the actual design. To overcome this problem PLM set up is used which provides safe sharing of information between the two without affecting the OEM's intellectual property rights. Second study shows that MRO vendor did not synchronized its system with OEM's product management system which results in inconsistent information flow about the part numbers, document numbers, reference procedures, working bill of material etc.

A. Kumar et.al. (2014) provided a technique for measurement and inspection of machine element using reverse engineering tool. Their study uses a spur gear which wears out during operation due to friction. The methodology includes scanning of worn out spur gear to obtain the point data, generation of surfaces and creating a 3D model, and final step is comparing the two model i.e. model obtained after scanning of worn out part to that of obtained from actual gear tooth profile so that worn area can be recognized and further action of maintenance can be applied.

A. Durupt et.al. (2008) have said that results obtained from current RE approaches are of no use if we consider the scope of re- design because the geometric models were either frozen or were not re-usable. So the main goal of their study was to provide a software tool which can be used to obtain a complete and fully parameterized CAD model including design objectives.

There are many more components which are reverse engineered in automobile industries such as mechanical seals, air conditioning system dryer housing assembly, valves, sprinkler valves, high temperature bolt/ washer assembly etc.

\subsection{Applications in software industries}

Reverse engineering has finds its way in software industries as well. RE here is a process of examination only i.e. the software under consideration is not modified. There are several reasons for which RE is used in software industries such as

1. Source code is available for the software but higher level aspects of the program are not properly documented or are no longer valid.

2. If the source code itself is not available for the software.

3. Security auditing.

4. Removal of copy protection.

5. To detect and neutralize viruses and malware.

E. J. Byrne (1991) experimented to reverse engineer a program. The experimentation was done in order to develop an Ada implementation of a FORTRAN program and upgrade the existing documentation. For this they extracted the design information from the FORTRAN source code and entered this information into a software development environment and then the extracted design information was used to implement a new version of the program written in Ada. During the study various issues was revealed concerning about the recovering design 
Rathore, N. \& Jain, P. K.: Reverse Engineering Applications in Manufacturing Indu... information such as separating design details from implementation details, incomplete or fallacious information, traceability of information between implementation and recovered design, and re-engineering.

\subsection{Applications in medical life sciences}

In medical life sciences reverse engineering plays a very important role in order save time and money, and to achieve higher accuracy of product. Improved medical production system comprises four main parts:

1. Measurement system to measure and capture human anatomy.

2. Software system to design the CAD model from the digitized data.

3. Advanced fabrication system in order to manufacture the part.

4. New materials for better fabrication than that were used in manual processes.

RE finds its usage in medical field in terms of following applications:

1. Applications in orthopedic, dental and reconstructive surgery.

2. Imaging modeling and replication of a patient's bone structure.

3. Models can be viewed and physically handled before surgery, which gives benefit during evaluation of the procedure and implementation in difficult cases.

4. Less risk to the patient had reduced cost through saving in theatre time.

Y. P. Lin et.al. (2005) to meet the customized needs of customer in short time interval and least cost have proposed a method to generate the complex surface of an artificial knee joint by using a CMM and digital model construction. They have also provided a plan for measurement so that better point data can be gathered. After the generation of point cloud data free form surfaces were constructed and then CAD model was developed. These models can be used as basis in which only local data needs to be changed for customized needs of different customers.

\subsection{Applications in film entertainment or animation industry}

Reverse engineering is used in film entertainment or animation industry in order to give following advantage:

1. Animated objects are imparted motion using the reverse engineered human skeletons.

2. For 3D scanning and rapid surfacing of scale models for animation and film sets.

3. For 3D scanning to support online marketing and e presentations.

4. For bringing real life forms into virtual gaming industry.

The one of the best example of use of reverse engineering application in film industry is shown in $86^{\text {th }}$ academy award winner movie named Gravity. Cinematographer Emmanuel Lubezki and VFX supervisor Tim Webber of Framestore, Cuaron (***, 2014) decided to make it almost entirely in CG (placing the actors' faces into the virtual environments with the only physical sets being the interiors of the two space 
capsules and portions of the ISS space station). For this they uses reverse engineering tool. Firstly they choreographed Gravity like an animated movie and then breaking the pieces apart and making them fit as needed. They have to go backwards and then forwards again to finesse the animation to quite a large degree before they started shooting it.

\section{Conclusion}

Reverse engineering is defined as the art of replicating an object, artifact or software with lost document in order to reveal the secrets of design, material, specifications or functions. Process of RE includes taking a system or component and then understanding its structural and functional aspects by analyzing the detailed $\mathrm{CAD}$ data of the component taken under consideration. Reverse engineering process involves digital measuring of component using digitized optical scanners (noncontact type) or CMM's (contact type) and then creating a 3D model from the point data obtained using $\mathrm{CAD} / \mathrm{CAM} / \mathrm{CAE}$ or other software's. $\mathrm{RE}$ is found to be an effective tool in case where design modifications have to be imparted in a product whose original CAD model is not available. It also plays a vital role in wear inspection processes of complex shapes which are if done manually are found to be very complex, time consuming and costly. It can also help in gaining competitive benchmarking because it allows understanding of rivalry's product design secrets. It also helps in medical industry as before the actual implementation of any artificial artefact inside the human body we can simulate the results of virtual model which results in life saving event and also saves time and money. Hence main objective of reverse engineering process is to reduce leads times so that the manufacturing time can be reduced automatically. Ability to reduce inspection time or lead time, reverse engineering approaches gaining popularity in today's competitive market because the current global environment is evolving at a rapid rate and seeking new ways to overcome time constraints and come up with new and improved product. But future work is also required in this field as there is a possibility of improvement in reverse engineering approaches as they lacks in efficiency and accuracy. Most of the Reverse engineering algorithms need further improvement for better result and less possibility of occurrence of error. Hence RE processes require further refinement so that one can use them for producing better quality products. Effective and efficient use of reverse engineering applications can enlarges the scope of its use in future.

\section{References}

Bagci, E. (2009). Reverse engineering applications for recovery of broken or worn parts and re-manufacturing: Three case studies. Advances in Engineering Software, Vol. 40, pp 407-418 
Rathore, N. \& Jain, P. K.: Reverse Engineering Applications in Manufacturing Indu...

Tsakatikas, D. And Kaisarlis, G. (2007). Reverse engineering in industrial maintenance component criticality analysis. $4^{\text {th }}$ International Conference on NDT, China, October and 2007, Hellenic Socitey for NDT, Greece

Abella, R. J.; Daschbach, J. M. and McNichols, R. J. (1994). Reverse engineering industrial applications. Computers and Industrial Engineering, Vol. 26, Issue 2, pp 381-385

Yau, H.T. (1997). Reverse engineering of engine intake ports by digitization and surface approximation. International Journal of Machine Tools and Manufacture, Vol. 37, No. 6, pp 855-871

Chang, M. and Park, S. C. (2008). Reverse engineering of a symmetric object. Computers and Industrial Engineering, Vol. 55, pp 311-320

Zhang, Y. (2003). Research into engineering application of reverse engineering technology. Journal of Materials Processing Technology, Vol. 139, pp 472-475

Sansoni, G. and Docchio, F. (2004). Three dimensional optical measurement and reverse engineering for automotive applications. Robotics and Computer-Integrated Manufacturing, Vol. 20, pp 359-367

Gao, J.; Chen, X.; Zheng, D.; Yilmaz, O. and Gindy, N. (2006). Adaptive restoration of complex geometry parts through reverse engineering application. Advances in Engineering Software, Vol. 37, pp 592-600

Wu, H.; Gao, J.; Li, S.; Zhang, Y. and Zheng, D. (2013). A review of geometric reconstruction algorithm and repairing methodologies for gas turbine components. International Journal of Telecommunication, Computing, Electronics and Control, Vol.11, pp 1609-1618

Lee, S. G.; Ma, Y. S.; Thimm, G. L. and Verstraeten, J. (2008). Product lifecycle management in aviation maintenance, repair and overhaul. Computers in Industry, Vol. 59, pp 296-303

Byrne, E. J. (1991). Software reverse engineering: A case study. Software: Practice and Experience, Vol. 21, issue 12, pp 1349-1364

Lin, Y. P.; Wang, C. T. and Dai, K. R. (2005). Reverse engineering in CAD model reconstruction of customized artificial joint. Medical Engineering \& Physics, Vol. 27, pp 189-193

Kumar, A.; Jain, P. K. And Pathak, P. M. (2014). Reverse engineering approach for measurement and inspection of machine element. International Journal of Advanced Manufacturing Systems, Vol. 15, issue 1, pp 95-101

Durupt, A.; Remy, S.; Ducellier, G. and Derigent, W. (2008). A new knowledge based approach the reverse engineering of a product. International Design Conference, Crotia, May and 2008, Design Information and Knowledge, Dubrovnik *** (2014) http://www.awn.com/vfxworld/reverse-engineering-gravity, Accessed on: 2014-10-17 